\title{
CÂNCER GÁSTRICO PRECOCE: CONTRIBUIÇÃO AO DIAGNÓSTICO E RESULTADO DO TRATAMENTO CIRÚRGICO
}

\author{
EARLY GASTRIC CANCER: CONTRIBUTION TO DIAGNOSIS AND RESULTS OF \\ SURGICAL TREATMENT
}

\author{
Cirilo Luiz Pardo Mêo Muraro, TCBC-SP ${ }^{1}$
}

\begin{abstract}
RESUMO: Objetivos:Analisar o diagnóstico e tratamento de pacientes portadores de câncer gástrico precoce. Método: Foram estudados 34 pacientes portadores de neoplasia gástrica precoce (CGP) tratados no Serviço de Cirurgia do Aparelho Digestivo Alto da Faculdade de Ciências Médicas da PUC- Campinas durante o período de janeiro de 1978 a dezembro de 1998. Resultados:O diagnóstico foi feito através da endoscopia digestiva alta e biópsia, sendo que em dois deles $(5,9 \%)$ a biópsia revelou apenas atipias em lesão ulcerada gástrica. O estudo histopatológico das peças cirúrgicas confirmou a existência de adenocarcinoma em todos os pacientes. A localização mais freqüente da neoplasia foi o antro gástrico e os tipos macroscópicos mais encontrados foram IIc e IIc + III. Quanto à profundidade na parede do estômago, verificou-se a prevalência da localização na mucosa. Em nenhum dos casos constatou-se invasão linfonodal. Todos os doentes foram submetidos à gastrectomia subtotal com linfadenectomia $\mathrm{D}_{2}$ e a reconstrução mais freqüente foi a gastrojejunostomia em Y de Roux (67,6\%). O seguimento pós-operatório dos pacientes variou de 17 dias a 21 anos e meio, o qual mostrou complicações em três deles (8,8\%). Apenas dois óbitos ocorreram $(5,8 \%)$, um por complicações pós-operatórias e outro, tardiamente, por recidiva da doença. Conclusões: A incidência de câncer gástrico precoce (CGP) encontrada no presente estudo $(8,7 \%)$, mostrou-se semelhante àquela referida pela literatura dos países ocidentais, sendo, entretanto, pobre em relação à incidência referida nas séries japonesas. Este fato valoriza a necessidade da realização de exames endoscópicos periódicos nos pacientes que compõem o chamado grupo de risco para a doença neoplásica do estômago. Finalmente, entendeu-se que quando o CGP está localizado na mucosa e não apresenta invasão de linfonodos e nem metástases à distância, a sobrevida é muito boa.
\end{abstract}

Descritores: Neoplasias gástricas; Gastrectomia; Diagnóstico; Cirurgia.

\section{INTRODUÇÃO}

O câncer gástrico é a mais freqüente das neoplasias malignas do aparelho digestivo e ocupa o segundo lugar entre todos os cânceres do homem e o quarto entre as mulheres. No sexo masculino é suplantado apenas pelos tumores de pele e, no feminino, pelos tumores de pele, colo de útero e mama. Representa $10 \%$ dos casos de neoplasia maligna entre os homens e 4\%, entre as mulheres. É mais freqüente no homem, na proporção de 2:1.

Tem incidência maior no Japão, seguido do Chile, Finlândia e Islândia, e menor nas Filipinas, Honduras e Estados Unidos. No Japão corresponde a $40 \%$ de todas as neoplasias malignas, com mortalidade de 45 casos em 100.000 habitantes. Nos Estados Unidos estes dados correspondem, respectivamente, a $5 \%$ e $8 \%$ dos casos. Estudos realizados

1. Professor Titular (Docente Livre) da Disciplina de Moléstias do Aparelho Digestivo I e Serviço de Cirurgia do Aparelho Digestivo Alto da Faculdade de Ciências Médicas da Pontifícia Universidade Católica de Campinas.

Recebido em 27/05/2002

Aceito para publicação em 02/09/2003

Trabalho realizado na Disciplina de Moléstias do Aparelho Digestivo I e Serviço de Cirurgia do Aparelho Digestivo Alto da Faculdade de Ciências Médicas da Pontifícia Universidade Católica de Campinas. 
no Brasil, no Estado de São Paulo, revelaram uma mortalidade de 17 casos da moléstia para cada 100.000 habitantes. Apesar do declínio da sua incidência em todo o mundo, há cerca de 650.000 casos, a cada ano ${ }^{1}$.

Embora de etiologia desconhecida, estudos epidemiológicos mostram a grande importância dos fatores dietéticos no seu desenvolvimento . A alta ingestão de alimentos defumados, de sais e condimentos e de alimentos fertilizados ou cozidos com substâncias carcinogênicas (nitritos, nitratos, benzopirenos etc.), também fazem parte deste conjunto que, quando associados a infecção por Helicobacter pylori, desencadeiam uma série de transformações que podem resultar no aparecimento de um adenocarcinoma.

Fatores hereditários e imunológicos também têm sido considerados na etiopatogenia da moléstia.

Descrito pela primeira vez em 1939 por Gutmann, o câncer gástrico precoce (CGP) é caracterizado como a neoplasia gástrica maligna cujo crescimento está limitado à mucosa (carcinoma intramucoso) ou submucosa (carcinoma submucoso) do estômago, com ou sem metástases em linfonodos regionais, segundo definição da Sociedade Japonesa de Endoscopia Digestiva em 1962. Ele é também denominado câncer gástrico incipiente, pré-invasivo ou in situ $^{2,3}$.

O diagnóstico do CGP tem sido feito em cerca de $50 \%$ dos casos no Japão, enquanto que nos países ocidentais estas cifras alcançam apenas $10 \%$. No Japão, este fato deve-se a inúmeras campanhas e programas, objetivando a detecção precoce do câncer do estômago. Este fato também é responsável pela absoluta falta de concordância entre os resultados da literatura japonesa e a dos outros países, quanto ao tratamento cirúrgico do câncer gástrico.

O presente trabalho tem por objetivos a análise dos aspectos diagnósticos e do resultado do tratamento cirúrgico empregado nos pacientes portadores de neoplasia gástrica precoce.

\section{MÉTODO}

Foram estudados 34 pacientes portadores de neoplasia gástrica precoce (CGP) tratados no Serviço de Cirurgia do Aparelho Digestivo Alto da Faculdade de Ciências Médicas da PUC- Campinas durante o período de janeiro de 1978 a dezembro de 1998.

\section{RESULTADOS}

Dezenove pacientes eram do sexo masculino $(55,8 \%)$ e 15 , do sexo feminino $(44,2 \%)$. Quanto à etnia, 27 eram brancos $(79,4 \%)$, seis negros, $(17,6 \%)$, e apenas um amarelo (2,9\%). A idade máxima foi 84 anos e a mínima 25 anos, com média de 57 anos. A localização mais frequiente foi no antro gástrico, o que ocorreu em 24 casos (70,6\%), vindo a seguir a incisura angularis e o corpo do estômago, com cinco pacientes $(15,1 \%)$ em cada uma destas regiões. Em nossos 34 pacientes, $31(91,2 \%)$ deles apresentavam epigastralgia, como o sintoma mais frequiente, seguido da dor no hipocôndrio direito plenitude pós prandial e emagrecimento (Gráfico 1). Todos os 34 pacientes, foram submetidos à endoscopia digestiva alta com biópsia. Os achados macroscópios revelaram: lesão ulcerada gástrica em sete $(20,6 \%)$, sendo que em apenas um destes pacientes a lesão tinha características malignas, ou seja, tinha contorno irregular e bordas elevadas. Vinte e quatro doentes $(70,6 \%)$ evidenciaram aspecto macroscópico compatível com carcinoma gástrico precoce, sendo que em dez $(29,4 \%)$, a lesão era tipo IIc + III, em 11(32,3\%) tipo IIc e em outros três deles $(8,8 \%)$, tipo I. Dois pacientes $(5,9 \%)$ apresentaram lesão polipóide, um $(2,9 \%)$ cicatriz de úlcera gástrica e em outro $(2,9 \%)$ a neoplasia era de coto gástrico (Gráfico 2). Trinta e dois doentes $(94,1 \%)$ tiveram seu diagnóstico pré-operatório de adenocarcinoma, enquanto que os dois restantes $(5,9 \%)$ apresentavam atipia de bordas na lesão ulcerada gástrica. Estes últimos estavam entre os seis com lesão ulcerada gástrica sem características de malignidade. $\mathrm{O}$ estadiamento pré-operatório foi realizado com os seguintes exames: RX de tórax, ultrassonografia ou tomografia computadorizada abdominal e exames laboratoriais. Estes últimos foram: fosfatase alcalina, g glutamil transferase (g GT), bilirrubinas e colesterol.

O estadiamento da neoplasia não revelou presença de metástases em qualquer dos nossos pacientes, o que verificou-se também pelo estadiamento intraoperatório. A cirurgia realizada em todos os pacientes foi a gastrectomia subtotal com linfadenectomia $\mathrm{D}_{2}$. Este tipo de cirurgia implica na remoção do $1^{\circ} \mathrm{e}$ $2^{\circ}$ grupos de linfonodos, ditos respectivamente de perigástricos e extra-perigástricos, além dos dois omentos e do ligamento hepato-duodenal. A referida remoção linfonodal compreende os linfonodos de 1 a 6 (Grupo 1) e de 7 a 11 (Grupo 2). 


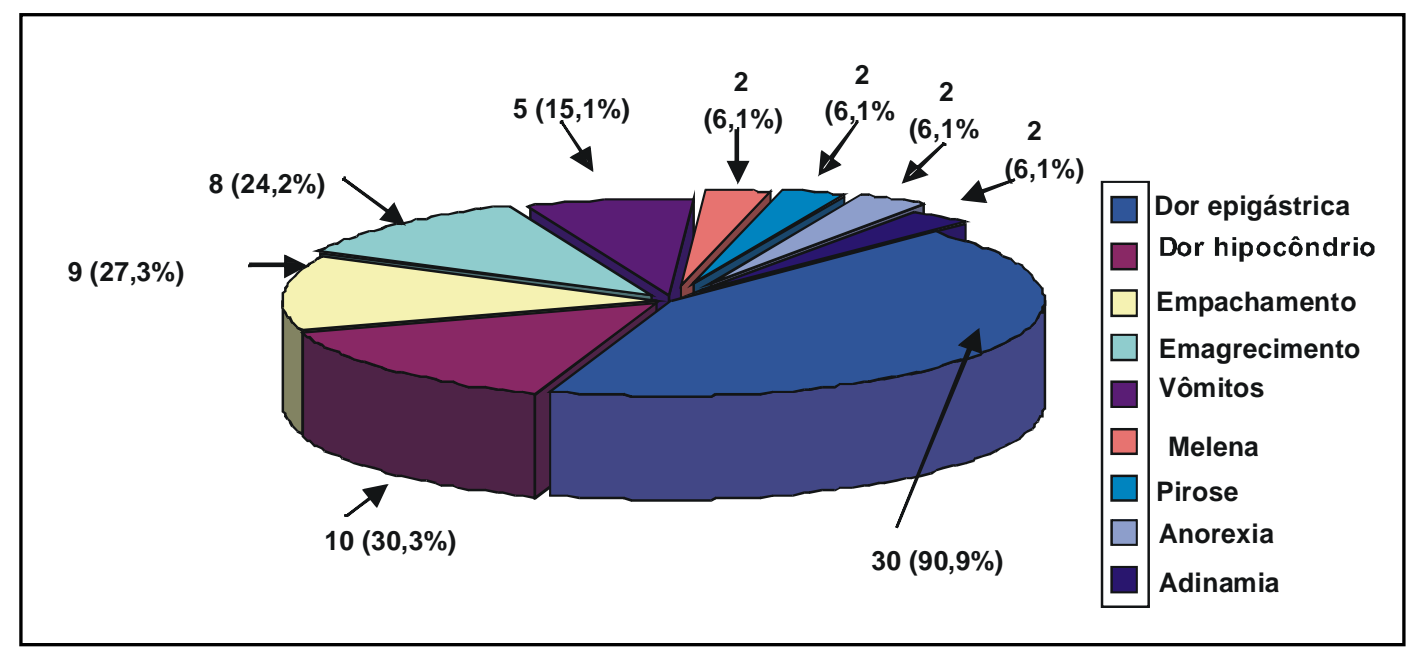

Gráfico 1 - Sintomatologia.

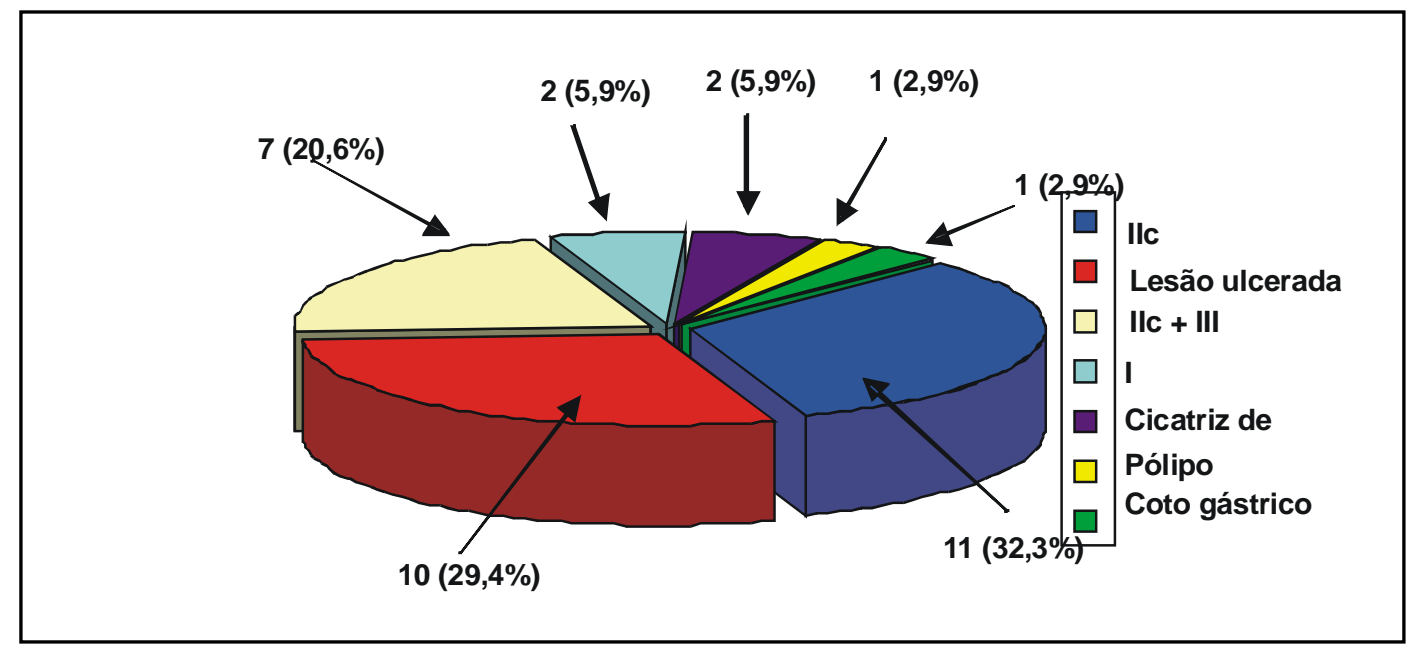

Gráfico 2 - Tipo (aspecto macroscópico) da lesão.

O estudo anátomo-patológico da peça cirúrgica demonstrou adenocarcinoma gástrico na mucosa em 22 pacientes $(64,7 \%)$ e na submucosa nos 12 restantes $(35,3 \%)$. Quanto ao tipo de reconstrução , a gastro-jejunostomia a Billroth II foi realizada em nove pacientes $(26,5 \%)$, Billroth I em dois $(5,9 \%) \mathrm{e}$ Y de Roux nos demais $23(67,6 \%)$. O paciente que apresentava neoplasia no coto gástrico, teve a sua reconstrução anterior à Billroth II convertida em Y de Roux, estando, portanto, entre estes últimos. Todas as cirurgias praticadas foram oralis parciais, pré-cólicas, com anastomoses junto à grande curvatura gástrica. Em vários casos utilizou-se sutura mecânica.
As complicações pós-operatórias compreenderam um caso de deiscência com fístula do coto duodenal, agravado por edema agudo do pulmão, resultando em óbito no $17^{\circ}$ dia após a cirurgia. Um paciente apresentou pneumonia lobar e um terceiro teve gastrite biliar de refluxo, sendo que os dois últimos tiveram evolução favorável. Assim, as complicações foram observadas em três pacientes $(8,7 \%)$, e o índice de mortalidade foi de $2,9 \% \mathrm{O}$ seguimento pós-operatório dos doentes variou de 17 dias a 21 avos e seis meses e se deu no terceiro e sexto meses no primeiro ano e anualmente, na maioria dos casos, nos anos subseqüentes. Apenas um paciente $(2,9 \%)$ da presente série, teve recidiva da doença, vindo a fale- 
cer em decorrência dela no terceiro ano de pós-operatório, com metástase hepática e carcinomatose peritoneal. A sobrevida de cinco anos foi observada em 22 dos nossos doentes $(97,4 \%)$, com relação aos doentes acompanhados por esse período, pois um dos pacientes faleceu por recidiva após três anos de pósoperatório. Dos 22 pacientes referidos, três deles $(8,8 \%)$ atingiram sobrevida de 10 anos; outros três $(8,8 \%) 15$ anos e um último paciente $(2,9 \%) 21$ anos e meio. Não estamos considerando aqui o paciente que faleceu de causa diferente da recidiva da neoplasia.

\section{DISCUSSÃO}

O câncer gástrico vem apresentando alguma redução de sua incidência nos últimos decênios. Apesar deste fato, permanece alta sua mortalidade ${ }^{1,4,5}$.

Quanto à incidência de CGP em relação aos cânceres gástricos diagnosticados, a diferença entre as estatísticas japonesas e as ocidentais são gritantes. Biasco et al ${ }^{6}$, estudaram 542 pacientes com câncer de estômago operados, e encontraram $15,3 \%$ de CGP. Endo $e t a l^{7}$, em 1.214 pacientes com câncer gástrico, conseguiram $31 \%$ de diagnóstico na fase precoce da moléstia.

Existem inúmeros trabalhos evidenciando a dificuldade do diagnóstico do CGP, pela existência de grande número de pacientes assintomáticos ou que apresentem quadro clínico atípico ${ }^{8-10}$. Lawrence et $a l^{11}$, estudando retrospectivamente 60 doentes com CGP, verificaram que $95 \%$ apresentavam quadro clínico atípico. Observaram também que 53,3\% deles foram tratados por 48 meses como se fossem portadores de doença benigna.

A dor epigástrica costuma ser, no entanto, o sintoma mais frequiente dos pacientes com CGP. Oleagoitia $e t a l^{9}$, ao analisarem 142 pacientes no período de dez anos, verificaram que a epigastralgia esteve presente em 75,3\% dos casos. Este fato tem sido confirmado por grande número de autores ${ }^{8-11}$. Outros sintomas como emagrecimento, anemia, plenitude gástrica, náuseas e vômitos, melena, fraqueza e anorexia, também têm sido relatados ${ }^{8,11,12}$.

O diagnóstico da moléstia é feito comumente através da endoscopia digestiva alta com biópsia. No entanto, alguns autores ${ }^{8-13}$ têm preconizado o estudo radiológico através da técnica de duplo contraste. Outros autore $\mathrm{s}^{14,15}$ têm utilizado de rotina, a ultrassonografia endoscópica na avaliação pré-operatória dos pacientes com câncer do estômago.
Folli et al $^{16}$, relataram $6,9 \%$ de falso-positivo com o estudo radiológico com duplo contraste, num grupo de 223 pacientes com CGP.

Todos os autores têm enfatizado a imperiosa necessidade da realização do diagnóstico precoce da doença, para melhorar o índice de sobrevida Nos países ocidentais o diagnóstico precoce ainda é bastante reduzido: cerca de 20\% nos Estados Unidos e 10\% na Europa.

No Japão o câncer gástrico precoce (CGP) tem cifras de $50 \%$ de detecção, devido aos métodos de investigação em massa (Stomach Mass Survey). Isto se deve à alta incidência da doença no país, à situação sócio-econômica privilegiada, e também à sofisticação dos equipamentos usados na propedêutica.

Ainda que no Brasil os métodos de investigação em massa sejam inviáveis, algo poderia ser feito neste sentido, desde que pacientes de alto risco para a doença fossem submetidos a exames endoscópicos rotineiros. Os serviços de saúde do país, mesmo os especializados, deveriam ser melhor orientados para conseguir a detecção da moléstia em sua forma inicial.

Além da maior frequiência do diagnóstico precoce, uma maior acuidade no estadiamento destes tumores no Japão tem contribuído para melhores índices de ressecabilidade e sobrevida. Outro fator importante para melhor êxito no tratamento destes doentes é, sem dúvida alguma, a padronização adequada dos procedimentos cirúrgicos, segundo relato de inúmeros trabalhos ${ }^{17}$, fato com o qual concordamos.

O diagnóstico do carcinoma gástrico precoce em relação ao câncer gástrico tem variado de $9 \%$ a $20 \%$ entre os autores de países ocidentais ${ }^{8,18}$, enquanto nas séries apresentadas pelos japoneses estas cifras se situam entre $40 \%$ e $50 \%$ 8,10,12,13,19-27.

Hayes et al $^{14}$, relatam sobrevida de 10 anos, ao redor de $90 \%$ em pacientes que não tinham metástases em linfonodos, enquanto que estas cifras caiam acentuadamente $(65 \%)$ nos doentes cujos linfonodos apresentavam-se invadidos pela neoplasia.

No que se refere à sobrevida de cinco anos quase todas as séries relatadas têm demonstrado cifras ao redor de $90 \%{ }^{8,10}$.

Folli et al ${ }^{16}$ na análise de 223 casos de CGP, relataram sobrevida de cinco anos em $91,7 \%$ deles, e de 10 anos em $87,8 \%$.

Inoue et $a l^{17}$, analisando grande casuística, relatam sobrevida de cinco anos de 73,2\% nos 
pacientes com CGP e demonstraram a importância da presença de metástase linfonodal e da profundidade do tumor na submucosa em relação com a sobrevida dos pacientes.

Vários autores ${ }^{10,17,28,29}$ têm referido uma incidência aproximadamente igual do CGP na mucosa e na submucosa, sendo que os índices de sobrevida apontados são melhores quando a neoplasia se localiza na mucosa gástrica.

Kitamura $e t a l^{28}$, fizeram estudo comparativo entre 582 pacientes sem acometimento de linfonodos e 52 com invasão linfonodal, mostrando que a incidência de invasão é maior quando a neoplasia precoce está localizada na submucosa do estômago. Estes autores observaram, portanto, que a invasão linfonodal está relacionada com a profundidade da lesão na parede gástrica; além disso, acreditam que o tamanho do tumor e a invasão dos vasos linfáticos também favoreçam a referida invasão. Por fim, enfatizam que os tipos macroscópicos e histológicos nada tem a ver com o acometimento dos linfonodos pela neoplasia gástrica precoce.

Jentschura et $a l^{30}$, verificaram, através do estudo de 1.310 doentes com CGP, que o tipo intestinal era mais freqüente que o tipo difuso da classificação de Lauren, na proporção de 60 para 40\%. Preconizam como margem de segurança na ressecção do estômago, $5 \mathrm{~cm}$ para o tipo intestinal e $10 \mathrm{~cm}$ para o difuso, pois estes últimos têm crescimento intramural.

O estadiamento pré-operatório tem sido considerado da maior importância por vários autores ${ }^{17,31}$ e devem constituir-se de exame físico, RX de tórax, ultrassonografia abdominal, exames laboratoriais, que incluem as enzimas de drenagem biliar, entre outros. Inoue et $a l^{17}$, enfatizam também a padronização do estadiamento da peça cirúrgica através de estudo histopatológico que deve procurar determinar a profundidade da invasão na parede gástrica, presença de linfonodos acometidos e tipo histológico da neoplasia.

O tipo de cirurgia preconizado para o CGP é a gastrectomia, subtotal ou total, dependendo da localização do tumor, com ressecção tipo $\mathrm{D}_{2}$, além da remoção dos dois omentos e do ligamento hepatoduodenal. Regueira et $a l^{32}$, acreditam que não existe diferença entre linfadenectomia $D_{1}$ e $D_{2}$, quanto à morbidade. Vários autores têm estudado a invasão linfonodal e preconizado a linfadenectomia adequada, correlacionando-a com a sobrevida ${ }^{17,29,33}$. Kitamura et al. ${ }^{28}$, acreditam que apenas $10 \%$ dos pacientes com
CGP devam ser submetidos à linfadenectomia, pois é este o percentual de invasão dos linfonodos pela doença. Adverte, porém, que é difícil a avaliação desta invasão, tanto no pré, quanto no intraoperatório. Propõem ressecção local nos casos sem invasão e com tumor intramucoso menor que $2 \mathrm{~cm}$ ou elevado menor que $1 \mathrm{~cm}$ e linfadenectomia $\mathrm{D}_{2}$ nos demais casos.

A recidiva após tratamento cirúrgico, embora pouco freqüente, tem sido relatada por alguns autores $^{15,18,34}$. A recorrência mais freqüente é no coto gástrico.

Guadagni et $a l^{35}$, em estudo multicêntrico relataram taxa de mortalidade operatória de 6,4\%, para o tratamento cirúrgico do CGP. Esses autores classificaram como diferenciadas as formas papilar e tubular do CGP e, como indiferenciados, os tipos adenocarcinoma pouco diferenciados e o de células em sinete. Os mesmos autores enfatizam a importância da linfadenectomia $\mathrm{D}_{2}$ e demontraram sobrevida de dez anos de 91,7\% com este tipo de ressecção. A linfadenectomia $\mathrm{D}_{3}$, manteve estas mesmas cifras de sobrevida, não sendo, assim, necessária. Já o estudo da sobrevida de 10 anos sem linfadenectomia, mostrou apenas $87,6 \%$ de sobrevida neste mesmo trabalho.

Todos os nossos pacientes foram submetidos à linfadenectomia $\mathrm{D}_{2}$, com margem de segurança superior a $5 \mathrm{~cm}$. A recidiva da neoplasia ocorrida em um dos nossos pacientes, esteve presente em um caso $(2,9 \%)$ em que o CGP localizava-se na submucosa do estômago. Yasuda et al ${ }^{36}$, observaram a importância do tamanho do tumor, correlacionando-o ao grau de invasão. Assim, propõem os seguintes tipos de dissecções linfonodais: $\mathrm{D}_{0}$ para tumores menores de 1 $\mathrm{cm}$., $\mathrm{D}_{1}$ para tumores entre $1 \mathrm{e} 4 \mathrm{~cm}$. e, finalmente, $\mathrm{D}_{2}$ para aqueles com diâmetro maior do que $4 \mathrm{~cm}$.

Outros métodos terapêuticos como a ressecção endoscópica através da injeção local de substâncias hipertônicas e a própria excisão local da lesão, têm sido preconizados ${ }^{12,28,37}$. O tratamento adjuvante, radioterapia ou quimioterapia, não tem demonstrado qualquer benefício para a sobrevida dos pacientes $^{8}$. A ressecção endoscópica tem sido assinalada por vários autores, como Tsujitani ${ }^{38}$, Yasuda ${ }^{38}$ e Tachibana ${ }^{39}$. A análise do nosso material e a revisão bibliográfica realizada permitem, no presente trabalho, as seguintes conclusões:

1. A incidência de CGP no nosso meio é pequena (cerca de 10\%), estando de acordo com o 
obtido pelo mundo ocidental e muito aquém das séries japonesas.

2. Uma melhor orientação dos serviços de saúde para o assunto, além de exames endoscópicos periódicos principalmente para doentes do chamado grupo de risco, deverão melhorar o prognóstico atual da moléstia.

3. Há a necessidade de se criar campanhas de profilaxia para o câncer de estômago, que devem incluir o aumento do número de diagnósticos precoces, além do tratamento adequado.
4. O estadiamento e a padronização cirúrgica adequados são fundamentais para a obtenção de bons resultados no tratamento do CGP.

5. A localização da neoplasia na mucosa gástrica, a ausência de linfonodos comprometidos e de metástases à distância, melhoram em muito a sobrevida obtida com o tratamento cirúrgico.

6. Os pacientes com patologias gástricas, incluindo-se a úlcera péptica, devem ser acompanhados através de exames endoscópicos periódicos mesmo no caso de lesões cicatrizadas.

\begin{abstract}
Background: To analysis diagnosis and treatment of patients with early gastric cancer. Methods: The author studied 34 patients with "Early Gastric Cancer" (EGC) treated at Faculdade de Ciências Médicas da Pontifícia Universidade Católica de Campinas from january 1978 to december 1998. Results: The diagnosis were performed by endoscopy and biopsy, but in two patients $(5,9 \%)$ the biopsy showed only atipic cells in gastric ulcer. In the other 32, biopsy showed adenocarcinoma. The most common localization of the neoplasia was in the gastric antrum and macroscopic types IIc and IIc + III predominated. The mucosal type appeared more often than the submucosal type and all patients had lymph nodes free of cancer invasion. All 34 patients underwent subtotal gastrectomy with D2 lymphadenectomy and in 67,3\% of them the Roux in $Y$ reconstruction was perfomed. Follow up ranged from 17 days to 21 years and six months and showed complications in only three cases $(8,8 \%)$. Two patients $(5,8 \%)$ died: one because of postoperative complications and the other because of tumor recurrence. Conclusions: EGC incidence in this study $(8,7 \%)$ is similar to that in the occidental countries literature, but is very low if compared to japanese series. These results emphasize the importance of periodic endoscopic examination of patients with risc factors for stomach neoplasic disease. When the CGP is limited to mucosa, lymph nodes are free and there are no distant metastasis, a very good survival is possible.
\end{abstract}

Key Words: Stomach neoplasms; Gastrectomy; Diagnostic; Surgery.

\section{REFERÊNCIAS}

1. Muraro CPM - "Câncer gástrico”. In Muraro CPM (eds) - Temas de cirurgia do aparelho alto. $1^{\text {a }}$ Edição. São Paulo. Fundo Editorial Byk, 2000, pp. 131-150.

2. Fielding WL, Ellis DJ, Jones BG, et al. - Natural history of "early" gastric cancer: results of a 10-year regional survey. Br Med J, 1980, 281(6246):965-967.

3. Zeni Neto C, Coelho JCU, Marchesini JB, et al. - Câncer gástrico precoce: apresentação de três casos e revisão da literatura. GED Gastroenterol Endosc Dig, 1996, 15(4):144-146.

4. Ichiyoshi Y, Toda T, Minamisono Y, et al. - Recurrence in early gastric cancer. Surgery, 1990, 107(5):489-495.

5. Kodama Y, Inokuchi K, Soejima K, et al. - Growth patterns and prognosis in ear gastric carcinoma. Superficially spreading and penetrating growth types. Cancer, 1983, 51(2):320-326.
6. Carter KL, Schaffer HA, Ritchie WP - Early gastric cancer. Ann Surg, 1984, 199(5):604-609.

7. Oleagoitia JM, Echevarria A, Santidrian JI et al. - Early gastric cancer. Br J Surg, 1986, 73(10):804-806.

8. Inoue $\mathrm{K}$, Tobe $\mathrm{T}$, Kan $\mathrm{N}$, et al. - Problems in the definition and treatment of early gastric cancer. $\mathrm{Br} \mathrm{J}$ Surg, 1991, 78(7): 818-821.

9. Hayes N, Karat D, Scott DJ, et al. - Radical lymphadenectomy in the management of early gastric cancer. Br J Surg, 1996, 83(10):14211423.

10. Lehnert T, Erlandson RA, DeCosse JJ - Lymph and blood capillaires of the human gastric mucosa. A morphologic basis for metastasis in early gastric carcinoma. Gastroenterology, 1985, 89(5):839-850.

11. Folli S, Dente M, Dell'amore D, et al. - Early gastric cancer: prognostic factors in 223 patients. Br J Surg, 1995, 82(7):952-956. 
12. Kitamura K, Yamaguchi T, Taniguchi H, et al: Analysis of lymph node metastasis in early gastric cancer: rationale of limited surgery. J Surg Oncol, 1997, 64(1):42-47.

13. Okamura T, Tsujitani S, Korenaga D, et al. Lymphadenectomy for cure in patientes with early gastric cancer and lymph node metastasis. Am J Surg, 1988, 155(3):476-480.

14. Biasco G, Paganelli GM, Azzaroni D, et al. - Early gastric cancer in Italy. Clinical and pathological observations on 80 cases. Dig Dis Sci, 1987, 32(2): 113-120.

15. Endo M, Habu H - Clinical studies of early gastric cancer. Hepatogastroenterology, 1990, 37(4):408-410.

16. 12. Bringaze WL, Chappuis CW, Correa P, et al. - Early gastric cancer. 21-year experience. Ann Surg, 1986, 204(2):103-107.

17. Gama-Rodrigues JJ, Bresciani CJ, Waisberg DL, et al. Surgical management of gastric carcinoma? Extent of gastric resection and lymphadenectomy - current trends and results. ABCD Arq Bras Cir Dig, 1986, 1(3):84-89.

18. Green PH, O’Toole KM, Slonim D, et al. - Increasing incidence and excellent survival of patients with early cancer: experience in a United States medical center. Am J Med, 1988, 85(5):658-661.

19. Guadagni S, Catarci M, Kinoshitá T, et al. - Causes of death and recurrence after surgery for early gastric cancer. World J Surg, 1997, 21(4):434-439.

20. Lawrence M, Shiu MH - Early gastric cancer. Twentyeight-years experience. Ann Surg, 1991, 213(4) 327 334.

21. Heesakkers JP, Gouma DJ, Thunnissen FB, et al. - Nonradical therapy for early gastric cancer. Br J Surg, 1994, 81(4):551-553.

22. Madden MV, Price SK, Learmonth GM et al. - Surgical stating of gastric carcinoma sources and consequences of error. Br J Surg, 1987, 74(2) 119-121.

23. Regueira FM, Hernández-Lizoain JL, Torramadé J et al. - Papel de la linfadenectomía ampliada tipo R2 en el tratamiento quirúrgico del cáncer gástrico resecable. Rev Esp Enferm Dig, 1994, 86(6): 884-890.

24. Seto Y, Nagawa H, Muto T - Impact of lymph node metastasis on survival with early gastric cancer. World J Surg, 1997, 21(2):186-190.
25. Hirao M, Masuda K, Asanuma T, et al. - Endoscopy resection of early gastric cancer and other tumors with local injection of hypertonic saline-epinephrine. Gastrointest Endosc, 1988, 34(3):264-269.

26. Tsujitani S, Oka S, Saito H, et al. - Less invasive surgery for early gastric cancer based on the low probability of lymph node metastasis. Surgery, 1999, 125(2):148-154.

27. Yasuda K, Shiraishi N, Suematsu T et al. - Rate of detection of lymph node metastasis is correlated with the depth of submucosal invasion in early stage gastric carcinoma. Cancer, 1999, 85(10): 2119-2223.

28. Tachibana M, Takemoto $\mathrm{Y}$, Monden $\mathrm{N}$ et al. Clinicopathological features of early gastric cancer: results of 100 cases from a rural general hospital. Eur J Surg, 1999, 165(4):319-325.

29. Jentschura D, Heubner C, Manegold BC, et al. - Surgery for early gastric cancer: a European one-center experience. World J Surg, 1997, 21(8):845-849.

30. Ichikura T, Uefuji K, Tomimatsu S, et al. - Surgical strategy for patients with gastric carcinoma with submucosal invasion. A multivariate analysis. Cancer, 1995, 76(6):935-940.

31. Mitsudomi T, Watanabe A, Matsusaka T, et al. - A clinocopathological study of synchronous multiple gastric cancer. Br J Surg, 1989, 76(3):237-240.

32. Muraro CPM, Alvarez GA, Aquino JLB, et al. - Carcinoma gástrico precoce - Análise de 18 casos. Anais Gastrão,1994, 9 (supl. 2): 43.

33. Muraro CPM, Aquino JLB, Lucena FTP, et al. - Correlação entre estadiamento e ressecabilidade no câncer gástrico. Rev Ciênc Méd PUCCAMP, 1997, 6 (2/3):8587.

34. Muraro CPM - Gastrite alcalina de refluxo. Estudo experimental sobre a etiopatogenia. Dissertação (Doutorado). Campinas. Unicamp, 1986.

35. Muraro CPM - Câncer gástrico precoce. Contribuição ao diagnóstico e resultado do tratamento cirúrgico. Dissertação (Livre Docência). Botucatu. Universidade Estadual Paulista, 2000.

Endereço para correspondência:

Cirilo Mêo Muraro

Av. Jesuíno Marcondes Machado, 357

13092-320 - Campinas - SP 